Thorax (1974), 29, 199.

\title{
Starch granules in the pericardium as a cause of the post-cardiotomy syndrome
}

\author{
M. P. O S B OR NE, M. PANETH, and K. F. W. H I N S N \\ Brompton Hospital, London
}

\begin{abstract}
Osborne, M. P., Paneth, M., and Hinson, K. F. W. (1974). Thorax, 29, 199-203. Starch granules in the pericardium as a cause of the post-cardiotomy syndrome. A case of the 'post-cardiotomy' syndrome following mitral valve replacement is reported. Recurring pericardial effusions and cardiac tamponade required surgical drainage. Pericardial biopsy demonstrated an inflammatory reaction in the presence of starch. It is suggested that starch might be one of several aetiological agents in the post-cardiotomy syndrome. Further investigation of such patients is indicated.
\end{abstract}

Over the past 30 years there has been considerable interest in the hazards of surgical glove powders. The use of lycopodium spores and magnesium silicate (talc) powder as surgical glove lubricants in the 1930s was followed by numerous reports describing the problems of foreign-body granulomata in surgical wounds and the peritoneal cavity (Antopol, 1933; Eiseman, Seelig, and Womack, 1947). Lee and Lehman (1947) described a non-irritating absorbable powder prepared from corn-starch which rapidly came into general use. The powder ('Biosorb', Ethicon Limited) is prepared from maize corn-starch and treated with epichlorhydrin to render it stable to autoclaving; $2 \%$ magnesium oxide and small amounts of sodium sulphate and sodium chloride are added to maintain the free-flowing properties. Early experimental studies in animals failed to demonstrate any adverse effects (MacQuiddy and Tollman, 1948). Several reports since have shown starch glove powder to be responsible for granulomatous lesions in the peritoneal cavity (McAdams, 1956; Paine and Smith, 1957; Neely and Douglas Davies, 1971). The manifestations of the post-cardiotomy syndrome, malaise, fever, pain, and inflammatory exudate with elevation of the erythrocyte sedimentation rate and normal leucocyte count are comparable to those of starch peritonitis. There have been no previous reports of starch pericarditis found after exhaustive search of the literature over the last decade.

\section{CASE REPORTS}

A 38-year-old Arab housewife was admitted to the Brompton Hospital on 27 February 1973 for investigation. She had presented with pain in the left upper chest posteriorly and her physician had heard a murmur. There was no history of exertional dyspnoea and her exercise tolerance was normal. She denied a history of rheumatic fever. She had not had any previous operation or recent medication and did not have any known allergies. She was fully active at home and had had three normal pregnancies.

On examination she presented the physical signs of fully developed critical mitral stenosis in sinus rhythm. There were no signs of cardiac failure.

INVESTIGATIONS $\mathrm{Hb} 10.9 \mathrm{~g} / 100 \mathrm{ml}$, normal WCC and differential; bloodfilm showed features of a microcytic hypochromic anaemia. ESR $5 \mathrm{~mm}$ in first hour.

A chest radiograph (PA) showed moderate left atrial enlargement with upper lobe blood diversion. No intracardiac calcification was seen. Echocardiography demonstrated thickening of the anterior cusp of the mitral valve with reduced diastolic closure rate but good amplitude of movement of the valve. The left ventricular cavity was of normal size. The presence of a left atrial myxoma was excluded.

OPERATION (9 March 1973) Through a median sternotomy an open mitral valvotomy was carried out, but as there was marked subvalvar chordal shortening resulting in an unacceptable degree of regurgitation, the value was exercised and replaced with a $29 \mathrm{~mm}$ tissue diameter Björk-Shiley (tilting-disc) prosthesis. 


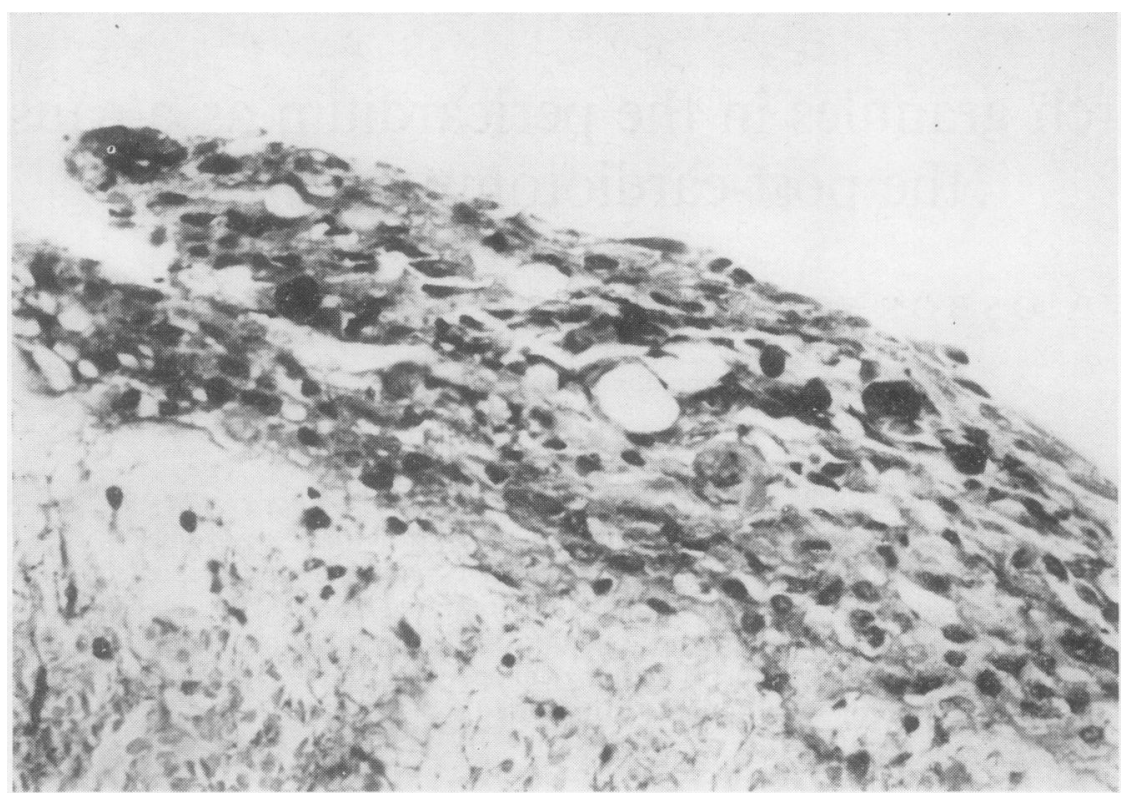

FIG. 1. Stained PAS showing the character of the exudate. The darkly staining bodies are $P A S$ positive granules.

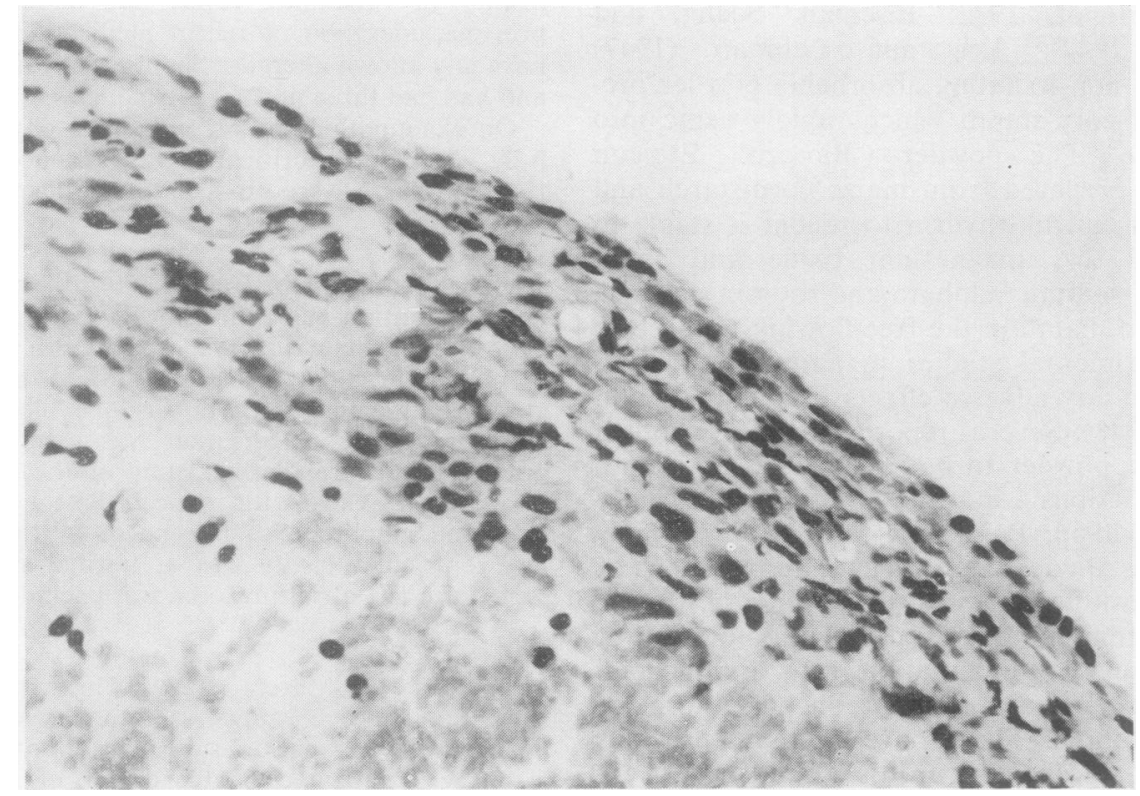

FIG. 2. Haematoxylin and eosin stain with partial polarization. 'Maltese-cross' figures becoming apparent. 


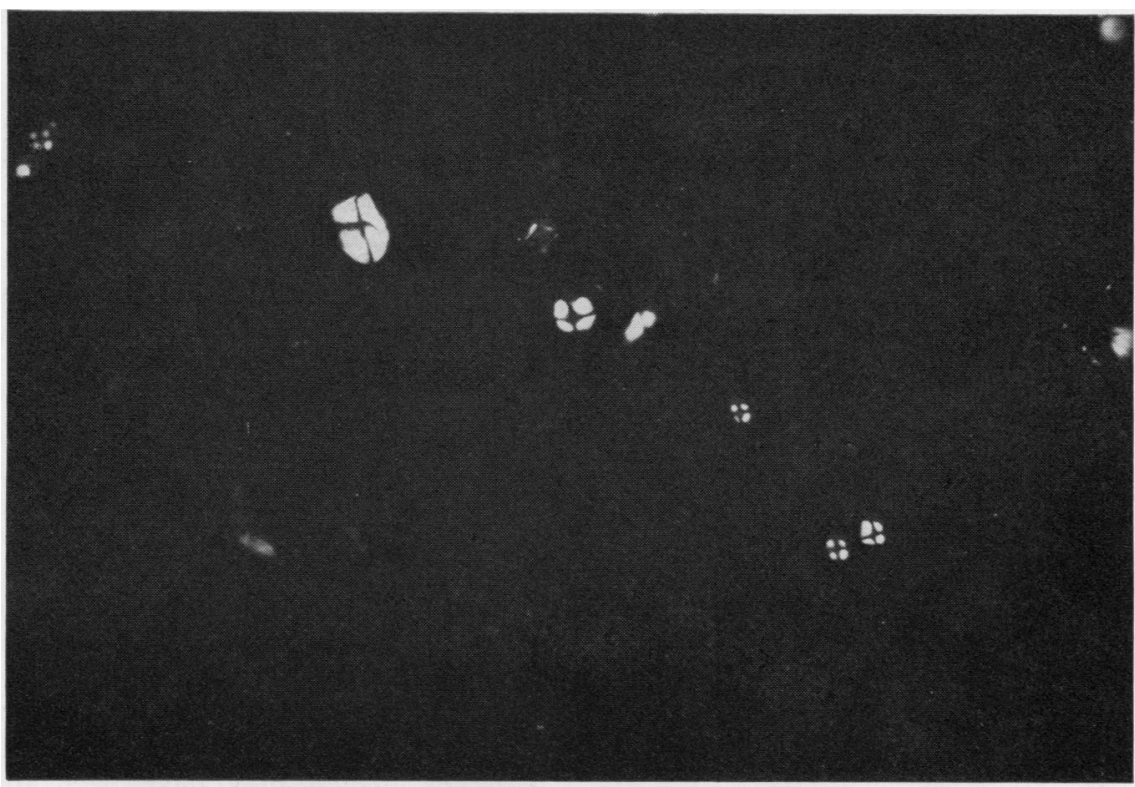

FIG. 3. Same field as in Figs. 1 and 2 with full polarization.

POSTOPERATIVE COURSE The patient made an uneventful immediate recovery. At the end of the first postoperative week there was a pyrexia of $37.5^{\circ}-38^{\circ} \mathrm{C}$ and she had a cough productive of mucopurulent sputum. She improved with physiotherapy and the routine course of postoperative antibiotics (cloxacillin, $500 \mathrm{mg}$ six-hourly, streptomycin sulphate, $0.5 \mathrm{~g} \mathrm{12}$-hourly). She was discharged on the twelf th postoperative day on dipyridamole (Persantin), $50 \mathrm{mg}$ orally three times daily.

Forty-eight hours later she was readmitted complaining of malaise, abdominal discomfort, nausea, and vomiting. She was apyrexial, not dyspnoeic or icteric; she had a sinus tachycardia and a blood pressure of $130 / 80 \mathrm{mmHg}$. The jugular venous pressure was elevated by $7 \mathrm{~cm}$ from the sternal angle. On auscultation the prosthesis was functioning normally and there was in addition a third heart sound. No pericardial friction rub was heard. The liver was enlarged and tender but there was no peripheral oedema. Soon after admission she became hypotensive with marked arterial and venous pulsus paradoxus.

A diagnosis of the post-cardiotomy syndrome with cardiac tamponade was made. Pericardial aspiration through the xiphisternal route produced $250 \mathrm{ml}$ of straw-coloured fluid with immediate relief of the cardiac tamponade. Over the course of the next two weeks the pericardial effusion recurred three times, requiring aspiration on each occasion to relieve tamponade. During this period she continued to have a low-grade fever although treatment with oral prednisone, $60 \mathrm{mg}$ daily, had been instituted. In spite of two attempts to provide continuous pericardial drainage with an indwelling Intra-cath, a fourth episode of tamponade developed.

OPERATION (10 April 1973) Through a left anterior thoracotomy the pericardial cavity was opened and $350 \mathrm{ml}$ of bloodstained pericardial fluid was evacuated. The pericardium looked inflamed and covered with a fibrinous exudate. No discrete lesions were seen and no accumulation of old blood-clot was found. A pleuropericardial window was made and a biopsy of the pericardium was taken. The incision was closed with a pericardial drain which was removed after 48 hours.

The patient initially made a good recovery but on the ninth postoperative day (19 April 1973) cardiac tamponade recurred. A chest radiograph revealed a left pleural effusion as well as an enlarged cardiac shadow. A wide-bore intercostal tube was inserted into the left pleural cavity and $540 \mathrm{ml}$ of strawcoloured fluid was drained under pressure. After 72 hours the drainage tube was removed and she was discharged on oral prednisone, $30 \mathrm{mg}$ daily. Steroid therapy was tailed-off after three weeks without further clinical relapse.

\section{INVִESTIGATIONS}

Haematological $\mathrm{Hb} 11.7 \mathrm{~g} / 100 \mathrm{ml}$, total WCC $10,500 / \mathrm{mm}^{3}, 5 \%$ eosinophils, ESR $20 \mathrm{~mm}$ in first hour. 
Biochemical Total serum proteins $6.4 \mathrm{~g} / 100 \mathrm{ml}$, serum albumin $3.4 \mathrm{~g} / 100 \mathrm{ml}$, serum globulin $3.0 \mathrm{~g} /$ $100 \mathrm{ml}$. Serum protein electrophoresis showed a slight increase in the $\gamma$-globulin fraction.

Bacteriological Culture of the pericardial aspirate was sterile.

Immunological Immunoglobulins-total $\quad 1 \quad \mathrm{~g} \quad \mathrm{G}$ $1235 \mathrm{mg} / 100 \mathrm{ml}$ (normal 500-1500); total $1 \mathrm{~g} \mathrm{~A}$ $450 \mathrm{mg} / 100 \mathrm{ml}$ (normal 125-450); total $1 \mathrm{~g} \mathrm{M} 266 \mathrm{mg}$ / $100 \mathrm{ml}$ (normal (50-170). Antinuclear factor negative, antiheart autoantibodies and antipericardial autoantibodies absent, serum precipitins to starch absent. Initial attempts to detect specific antistarch $1 \mathrm{~g} \mathrm{E}$ by the radio-allergosorbent (RAST) test (Wide, Bennich, and Johansson, 1967) were negative. An intradermal starch sensitivity test using $0.1 \mathrm{ml}$ of a $1 / 30$ dilution of a suspension of Biosorb glove powder in $\mathbf{N}$ saline injected intradermally, with a control of $\mathrm{N}$ saline, showed neither early nor late reactions (the patient was receiving steroids at this time).

Histological Haematoxylin and eosin stained sections of pericardium showed an inflammatory cellular exudate surrounding amorphous material on the surface of the pericardium. The inflammatory cells consisted of numerous lymphocytes with occasional eosinophils and histiocytes. PAS stain showed darkly staining clumps of carbohydrate (Fig. 1). Partial and fully polarized light microscopy showed that the carbohydrate masses exhibited with typical 'Maltese-cross' birefringence of starch granules (Figs. 2 and 3). A histological diagnosis of starch pericarditis was made.

\section{DISCUSSION}

Starch pericarditis following cardiac surgery has not been described. Following the introduction of starch powder as an absorbable non-irritating glove lubricant there have been numerous reports of granulomatous peritonitis resulting from its use. Extraperitoneal sites have been described, such as in surgical wounds (Sneierson and Woo, 1955), in the epididymis following scrotal surgery (Ising, 1960), and in the middle ear after stapedectomy (Rock, 1967). It was suggested by Bates (1965) and Taft, Lasersohn, and Hill (1970) that there should be no reason why a reaction to starch could not occur in another serous cavity such as the pericardium following surgery.

The patient had a constitutional illness with malaise, fever, pain, and recurring pericardial effusions three weeks after mitral valve replacement typical of the post-cardiotomy syndrome. Biopsy of the pericardium showed the typical 'Maltese-cross' birefringence of starch, when viewed with polarized light microscopy, sur- rounded by lymphocytes, occasional eosinophils and histocytes. Epithelioid cells and multinucleate giant cells described in typical starch granulomate (Bates, 1965) were not seen in this patient. The finding of starch granules in a serous cavity fol 8 lowing surgery does not necessarily mean than they have provoked an inflammatory reaction. (Swingler, 1971). Postlethwait, Howard, an $P$ Schanher (1949) have shown in experimenta $\vec{f}$ animals that starch is completely absorbed frond the pericardial cavity after surgery in 14 to 28 days, but the formation of starch granulomatio can occur. There is also experimental evidence that the presence of a foreign-body or infection (Lee, Collins, and Largen, 1952) and serosa 6 damage (Jagelman and Ellis, 1973) may delayo the absorption of starch and increase the likeli= hood of granuloma formation. Holgate, Wheeler 3 and Bliss (1973) have shown that a hyper $\frac{0}{4}$ sensitivity reaction to starch may be involved an 8 a cell-mediated immune reaction can occur. In $\overrightarrow{0}$ our patient a non-specific change in the immuno- $\rightarrow$ globulins, which may have been the result of cardiac surgery per se, and a negative skire sensitivity test were found. The skin reaction to starch may have been suppressed by the adminis tration of steroids (Bates, 1965). Care must be taken in skin sensitivity testing since an acute exacerbation of the condition may occur. Ouro patient did not respond to steroids alone but eventually made a good recovery, steroids being withdrawn without exacerbation. Starch peritonitis. has been successfully treated with steroids (Maggs and Reinus, 1959).

The aetiology of the post-cardiotomy syndrome is in doubt, and it has been suggested that it mayg be a reactivation of rheumatic fever (Soloff et al. 1953), traumatic due to surgical procedures (Wood, 1954), due to the presence of blood clot in the pericardial cavity (Ito, Engle, and Goldberg,, 1958) or an auto-immune process (van der Geld $\frac{D}{\mathrm{O}}$ 1964). We suggest that these cases should be investigated for the presence of starch pericarditis o by examination with polarized light microscopy of pericardial biopsy, if available, or a preparation of the pericardial fluid by the technique of $\omega$ Warshaw (1972) described for ascitic fluid. Skin sensitivity testing, immunological studies such as으 lymphocyte transformation, macrophage migration studies, and identification of humoral antibodies may be of value. It would be interesting to $\frac{T}{\circ}$ investigate further the possibility of starch acting as an adjuvant with damaged pericardium or $\frac{\mathcal{O}}{\mathbb{\Phi}}$ myocardium, thereby initiating an auto-immune $\varrho$ reaction. 
The problems of starch powder can be avoided either by finding a suitable alternative or by not having the outer surface of surgical gloves coated with powder (there is $0.562 \mathrm{~g}$ of powder on the outer surface of four pairs of gloves (Lee and Lehman, 1947). The preoperative washing of the gloves is not effective in removing all powder and results in clumping with consequent delay in absorption and a greater likelihood of granuloma formation (Jagelman and Ellis, 1973).

The post-cardiotomy syndrome was first described in 1953, starch glove powder being introduced generally at this time. This may be coincidental with the increasing volume of cardiac surgery. Starch pericarditis may be only one of the factors involved in the aetiology of the postcardiotomy syndrome.

Our thanks are due to Dr. R. V. Gibson, under whose care this patient was admitted, Professor Margaret Turner-Warwick, for carrying out immunological studies, and Mr. K. Moreman, of the Chester Beatty Institute, for the photomicrographs.

\section{REFERENCES}

Antopol, W. (1933). Lycopodium granuloma. Archives of Pathology, 16, 326.

Bates, B. (1965). Granulomatous peritonitis secondary to corn starch. Annals of Internal Medicine, 62, 335.

Eiseman, B., Seeling, M. G., and Womack, N. A. (1947). Talcum powder granuloma: a frequent and serious postoperative complication. Annals of Surgery, 126, 820.

Holgate, S. T., Wheeler, J. H., and Bliss, B. P. (1973). Starch peritonitis: an immunological study. Annals of the Royal College of Surgeons of England, 52, 182.

Ising, U. (1960). Foreign-body granuloma resulting from the use of starch glove powder at surgery. Acta Chirurgica Scandinavica, 120, 95.

Ito, T., Engle, M. A., and Goldberg, H. P. (1958). Postpericardiotomy syndrome following surgery for nonrheumatic heart disease. Circulation, 17, 549.

Jagelman, D. G. and Ellis, H. (1973). Starch and intraperitoneal adhesion formation. British Journal of Surgery, 60, 111.
Lee, C. M. and Lehman, E. P. (1947). Experiments with non-irritating glove powder. Surgery, Gynaecology and Obstetrics, 84, 689.

—, Collins, W. T., and Largen, T. L. (1952). A reappraisal of absorbable glove powder. Surgery, Gynaecology and Obstetrics, 95, 725.

Maggs, R. L. and Reinus, F. Z. (1959). Peritonitis, caused by surgical glove starch powder, treated with steroids. American Journal of Surgery, 98, 111.

McAdams, G. B. (1956). Granulomata caused by absorbable starch glove powder, Surgery, 39, 329.

MacQuiddy, E. L. and Tollman, J. P. (1948). Observations on an absorbable glove powder to replace talc. Surgery, 23, 786.

Neely, J. and Douglas Davies, J. (1971). Starch granulomatosis of the peritoneum. British Medical Journal, 3, 625.

Paine, C. G. and Smith, P. (1957). Starch granulomata. Journal of Clinical Pathology, 10, 51.

Postlethwait, R. W., Howard, H. L., and Schanher, P. W. (1949). Comparison of tissue reaction to talc and modified starch glove powder. Surgery, 25, 22.

Rock, E. H. (1967). Surgeon's glove powder (starch) middle ear granuloma. Archives of Otoloryngo$\log y, 86,8$.

Sneierson, H. and Woo, Z. P. (1955). Starch powder granuloma: A report of two cases. Annals of Surgery, 142, 1045.

Soloff, L. A., Zatuchni, J., Janton, O. H., O'Neil, T. J. E., and Glover, R. P. (1953). Reactivation of rheumatic fever following mitral commissurotomy. Circulation, 8, 481.

Swingler, G. R. (1971). Starch granulomatosis of the peritoneum. British Medical Journal, 4, 116.

Taft, D. A., Lasersohn, J. T., and Hill, L. D. (1970). Glove starch granulomatous peritonitis. American Journal of Surgery, 120, 231.

van der Geld, H. (1964). Anti-heart antibodies in the postpericardiotomy and the postmyocardialinfarction syndromes. Lancet, $2,617$.

Warshaw, A. L. (1972). Diagnosis of starch peritonitis by paracentesis. Lancet, $2,617$.

Wide, L., Bennich, H., and Johansson, S. G. O. (1967). Diagnosis of allergy by an in vitro test for allergen antibodies. Lancet, 2, 1105.

Wood, P. (1954). An appreciation of mitral stenosis. British Medical Journal, 1, 1113.

Requests for reprints to: M. Paneth, F.R.C.S., Brompton Hospital, London SW3. 